\title{
Low-level copper concentration measurements in silicon wafers using trace-element accelerator mass spectrometry
}

\author{
F. D. McDaniel, ${ }^{\text {a) }}$ S. A. Datar, B. N. Guo, and S. N. Renfrow \\ Ion-Beam Modification and Analysis Laboratory, Department of Physics, University of North Texas, \\ Denton, Texas 76203
}

Z. Y. Zhao

Varian IIS, Gloucester, Massachusetts 01930

J. M. Anthony

Texas Instruments, Inc., Dallas, Texas 75265-5936

(Received 30 December 1997; accepted for publication 4 April 1998)

\begin{abstract}
Accelerator mass spectrometry (AMS) is now widely used in over 30 laboratories throughout the world to measure ratios of the abundances of long-lived radioisotopes such as ${ }^{10} \mathrm{Be},{ }^{14} \mathrm{C},{ }^{36} \mathrm{Cl}$, and ${ }^{127} \mathrm{I}$ to their stable isotopes at levels as low as $10^{-16}$. Trace-element AMS (TEAMS) is an application of AMS to the measurement of very low levels of stable isotope impurities. Copper concentrations as low as 1 part per billion have been measured in silicon wafers. In this letter, we demonstrate the use of TEAMS to measure previously unknown copper concentration depth profiles in As-implanted Si wafers at a few parts per billion. To verify the TEAMS technique, the samples from the same wafer were measured with secondary ion mass spectrometry, which showed the same profiles, albeit plateauing out at a concentration level six times higher than the TEAMS measurement. The ability to measure at these levels is especially significant in light of the recent moves towards the use of copper interconnects in place of aluminum in integrated eircuits. (C) 1998 American Institute of Physics. [S0003-6951(98)00823-7]
\end{abstract}

Since its modern day inception in 1977, ${ }^{1,2}$ accelerator mass spectrometry (AMS) has found a wide variety of applications in archaeology, geology, environmental sciences, hydrology, etc. ${ }^{3}$ The sensitivity of AMS is extraordinary with the ability to measure radioisotope/stable isotope ratios as low as $10^{-16}$. $^{3}$

The measurement of stable trace isotopes is a natural extension of AMS. In particular, the ability to measure trace amounts of impurities in semiconductor materials would be very useful, since device performance is affected by impurities at the part per billion (ppb) level. ${ }^{4}$

The use of AMS for dopant detection in semiconductors was first reported in $1985 .{ }^{5}$ Recently, there has been growing interest in the use of trace-element AMS (TEAMS) as a complement to secondary ion mass spectrometry (SIMS) and dedicated TEAMS facilities are being constructed. The IonBeam Modification and Analysis Laboratory (IBMAL) at UNT, in conjunction with Texas Instruments Inc. (TI) has built such a system. ${ }^{6}$

The trace-element AMS system is essentially a negativeion magnetic sector SIMS system joined to an AMS beamline (Fig. 1). In the UNT TEAMS system, a negative-ion source is followed by a $90^{\circ}$ sector magnet, which injects negative ions into a $3 \mathrm{MV}$ tandem accelerator. The tandem accelerator is followed by a $40^{\circ}$ analyzing magnet $(\mathrm{mv} / \mathrm{q}$ analysis) and a $45^{\circ}$ double-focusing electrostatic analyzer ( $E / q$ analysis). In addition, at the end of the beamline, the ion energy is measured with either a silicon surface-barrier detector or an ionization chamber. An electrostatic quadru-

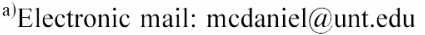

pole doublet is located just after the tandem accelerator and before the $40^{\circ}$ magnet. The system is designed for point-topoint focusing and the electrostatic quadrupole ensures that the tuning is mass independent. All the power supplies, controlling the various analyzing and focusing elements in the beamline, are under computer control and can be rapidly switched to sequentially transmit different mass ions through the TEAMS system. Measuring the energy of the ions at the end of the beamline ensures that any remaining mass/charge $(m / q)$ degeneracies (e.g., ${ }^{56} \mathrm{Fe}^{4+}$ and ${ }^{28} \mathrm{Si}^{2+}$ ), after passage through the magnetic sector and electrostatic fields, are resolved. Isobaric interferences can be eliminated by measuring a different isotope, since apart from In, every element has at least one stable isotope with no stable isobars.

The primary difference from a conventional radionuclide AMS system is the ion source. The TEAMS system at UNT

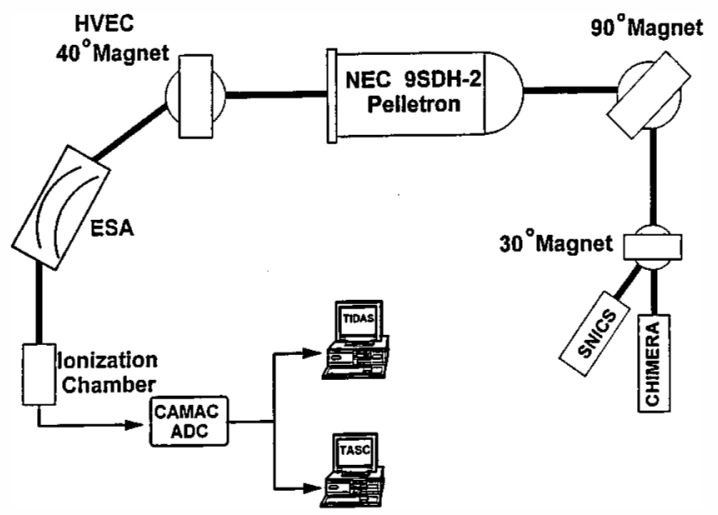

FIG. 1. Experimental apparatus of the TEAMS system in the Ion-Beam Modification and Analysis Laboratory at UNT. 
has an ultraclean ion source ${ }^{7}$ designed specifically to reduce contamination from metallic impurities. A key feature of the ion source is magnetic analysis of the primary (cesium) sputter beam reducing contamination from the primary beam. In addition, specially designed focusing and steering elements were made with ultrapure silicon surfaces, reducing impurities arising from the resputtering of beamline components. An electrostatic deflector, located in the secondary stage, ensures that the secondary ion beam injected into the accelerator is energy resolved, thereby reducing the tails from neighboring isotopes.

The negative-ion source is basically a SIMS ion source; the ion source and $90^{\circ}$ injection magnet effectively constitute a negative-ion magnetic sector SIMS system. ${ }^{8}$ Currently, SIMS is one of the most sensitive techniques for impurity determination in semiconductors, particularly for bulk and depth profile measurements. The main limitation of SIMS arises from molecular interferences, where the nominal mass of a secondary molecular ion is identical to that of the element being measured, e.g., ${ }^{30} \mathrm{SiH}$ has the same nominal mass as ${ }^{31} \mathrm{P}$ and ${ }^{28} \mathrm{Si}_{2}$ interferes with ${ }^{56} \mathrm{Fe}$. This becomes more of a problem as the atomic mass increases and the number of possible interferences increases. There are ways to reduce these molecular interferences in SIMS, but they require reduced system transmission, and therefore, reduced sensitivity. Virtually all molecular ions with charge state $3+$ or more breakup in the tandem accelerator. ${ }^{9}$ The molecular fragments are then removed by the magnetic and electrostatic analyzers following the accelerator. However, only one of the numerous charge states produced in the accelerator can be transmitted to the end of the beamline. Therefore, since only about $10 \%$ of the injected beam is transmitted to the end of the beamline, a reduction in sensitivity might be expected. This is offset by the fact that due to the much higher energy of the particles at the end of the line, even single particles can be measured with essentially $100 \%$ efficiency in either an ionization chamber or a solid-state detector. The dark current of either detector is practically zero, thus the lowest measurable count rate is determined by the counting time, which in turn is determined by the volume of available material. For bulk concentration measurements, this time can be very large, leading to detection limits approaching those of conventional AMS. In contrast, SIMS instruments use detectors which have a lowest detectable count-rate limit of about 0.5 counts per second (cps). For depth profile measurements, of course, the detection limit represents a compromise between depth resolution and sensitivity. The measured count rate represents the average concentration over a slice of material of a certain thickness, which in turn is determined by the counting time.

One can make an estimate of the sensitivity of the TEAMS system. Assume identical probabilities for negativeion formation (matrix and impurity) in the source and identical transmission through the beamline (this includes charge state fractionation occurring in the stripping process at the terminal of the tandem accelerator). Ignoring background contributions as well as variable isotopic abundances, an impurity count rate of $0.04 \mathrm{cps}$ with a charge state $3+$ matrix ion current of $5 \mathrm{nA}$ corresponds to an impurity/matrix ratio of approximately $4 \mathrm{ppt}$ with a depth window of $50 \AA$. A

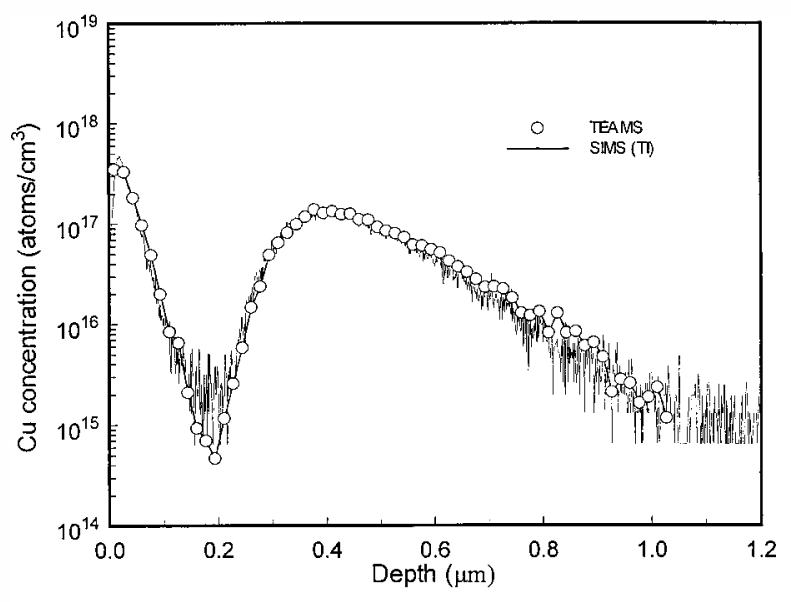

FIG. 2. Impurity copper concentration depth profile for an $80 \mathrm{keV}$, $1 e 16$ atoms $/ \mathrm{cm}^{2}$ As implant in Si. The TEAMS data (open circles) agree with the SIMS data very well. At the minimum near $0.2 \mu \mathrm{m}$, the TEAMS data show improved sensitivity by about a factor of 6 .

matrix current of $5 \mathrm{nA}$ at the end of the TEAMS beamline is readily achieved for a silicon sample with a $\mathrm{Cs}^{+}$primary current of $900 \mathrm{nA}$ at $10 \mathrm{keV}$ and a sample bias of $-11.5 \mathrm{kV}$. The cesium beam impacts the sample with an energy of 21.5 $\mathrm{keV}$ at an angle of $30^{\circ}$ corresponding to a penetration depth of around $140 \AA$ using the formula given in Ref. 10 .

Practically, the detection limit is determined by the background and negative-ion formation probability. Stable elements, especially relatively common ones such as $\mathrm{Fe}, \mathrm{Ni}$, $\mathrm{Cu}, \mathrm{Cr}$, etc., are often present in the materials which make up the source and beamline components in significant concentrations. The system can be the source of high background. The ultraclean source is designed to reduce such contamination.

Negative-ion formation probabilities vary widely and some atoms such as $\mathrm{N}$ and $\mathrm{Mn}$ do not form atomic negative ions at all. It would appear that this would make it impossible to measure these species. However, in almost all cases, one can find a suitable molecular ion $X M^{-}$where $X$ is the species to be measured and $M$ is either a matrix atom or Cs. In some cases, the molecular ion has a higher yield than the atomic ion. For the few elements which do not form molecular negative ions with a reasonable yield, such as the noble gases, positive SIMS is probably a better alternative, but then a tandem accelerator cannot be used.

The ability of the UNT TEAMS facility to successfully measure depth profiles for a variety of implants $(\mathrm{B}, \mathrm{P}, \mathrm{As}$, $\mathrm{Cu}, \mathrm{Ni}$ ) in silicon has already been demonstrated. ${ }^{11,12}$ Figures 2, 3, and 4 show depth profiles of impurity $\mathrm{Cu}$ in silicon wafers implanted with As. The TEAMS results were later confirmed using SIMS since the deep secondary peaks in the depth profiles were quite unexpected. Figures 2, 3, and 4 also show the results of SIMS measurements. The SIMS measurements were made on a CAMECA $4 \mathrm{f}$ at TI. Although As profiles were also measured at the same time, only the copper profiles are shown in the interest of clarity. Copper impurity detection has assumed greater importance due to the recent development of copper interconnects in integrated circuit manufacture. Otherwise, copper is very much an unwanted impurity as it is a fast-diffusing contaminant causing junction-leakage-current problems. ${ }^{13}$ 


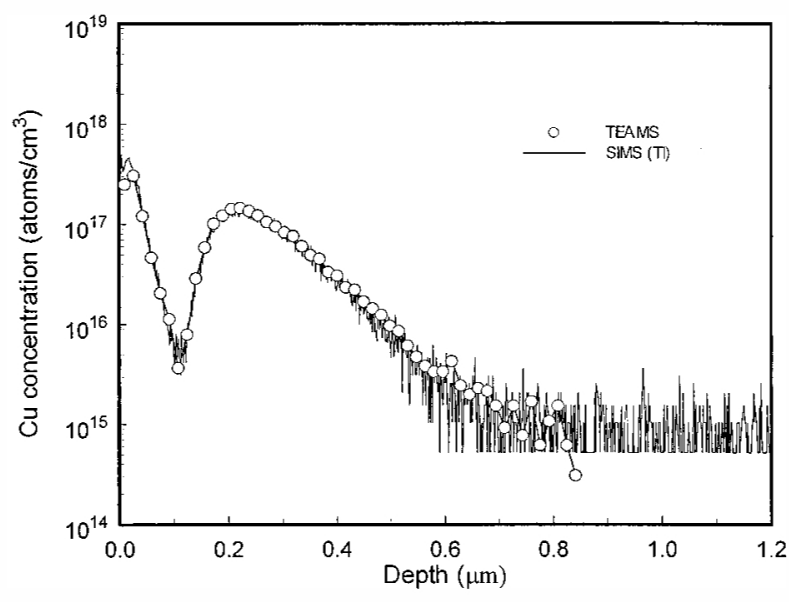

FIG. 3. Impurity copper concentration depth profile for a $40 \mathrm{keV}$, $1 \mathrm{e} 16$ atoms $/ \mathrm{cm}^{2}$ As implant in Si. Once again the TEAMS and SIMS data agree very well throughout the entire profile and the TEAMS data show improved sensitivity at the minimum concentrations.

For the TEAMS measurements, ${ }^{63} \mathrm{Cu}$ count rates were measured as a function of time. Conversion of the raw data to concentration versus depth profiles was done using ${ }^{65} \mathrm{Cu}$ implant standards. ${ }^{8}$ Integrating the depth profile for the standard implant gives the yield, since the total implanted dose is known for the standard. The yield can then be used to normalize the concentration measurements in the unknown samples. ${ }^{65} \mathrm{Cu}$ was used in the standard implant since it is the basis for the SIMS copper standard. Measurements were made with a sample bias of $11.5 \mathrm{kV}$ and $13.5 \mathrm{keV}$ and accelerator terminal voltage of $1.7 \mathrm{MV} .{ }^{63} \mathrm{Cu}^{3+}$ ions were counted at the end of the beamline in an ionization chamber. The cesium current incident on the samples was $800 \mathrm{nA}$. The beam spot was approximately $200 \mu \mathrm{m}$ in diameter and the crater was around $1.5 \mathrm{~mm}$ square. A digital spiral meander pattern was used for rastering the cesium beam across the sample and the ion counting was gated on the position of the cesium beam to accept data from the center region ( $12 \%$ plus beamwidth) of the crater. The sputtering rate was approximately $2.6 \AA / \mathrm{s}$ and the crater depth was measured with a

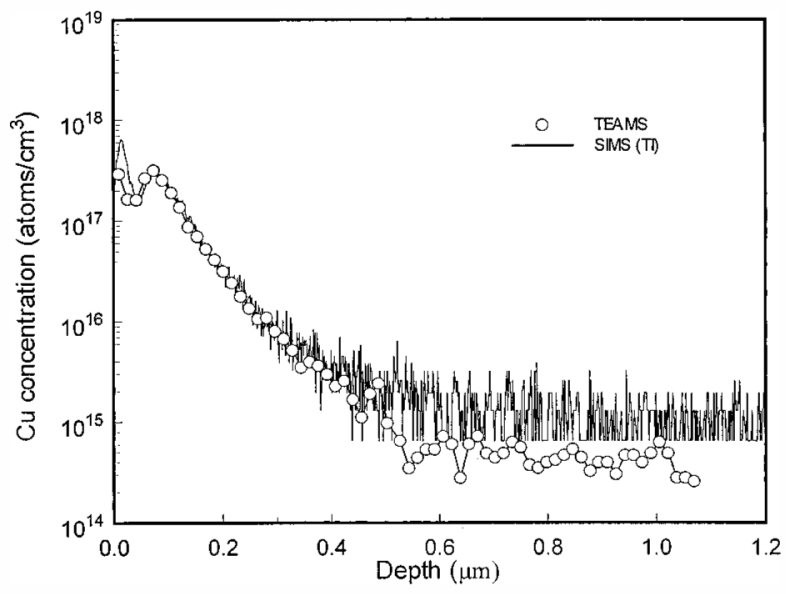

FIG. 4. Impurity copper concentration depth profile for a $10 \mathrm{keV}$, 1 el6 atoms $/ \mathrm{cm}^{2}$ As implant in Si. The TEAMS data points (open circles) agree very well with the SIMS data (solid line) down to a depth of $0.4 \mu \mathrm{m}$. Below that depth, the TEAMS profile shows improved sensitivity by about a factor of 6 . profilometer to ascertain the exact crater depth for each sample.

The agreement between the TEAMS and the SIMS data is very good. TEAMS is able to follow the copper profile down to below the $1 e 15$ atoms $/ \mathrm{cm}^{3}$ level, where the SIMS data are fuzzy. This is especially clear in Fig. 4. TEAMS measurements were also made with both isotopes of copper. The depth distribution for both isotopes was identical and the count rate scaled with the abundance. Thus, it is unlikely that the copper was somehow introduced into the samples as part of the As beam from the implanter, especially since the two isotopes differ by $2 \mathrm{amu}$. The correspondence between the depth distribution of $\mathrm{Cu}$ and the As-implant energy is under investigation.

The results show that TEAMS can successfully measure a priori unknown depth profiles. Thus, TEAMS can be usefully applied to solving certain problems in the processing of semiconductor materials, notably, ion implantation, In the meantime, work is underway to further improve the sensitivity. Improved calibration of the analyzing components and better alignment of the beamline should improve the transmission, thereby enhancing the sensitivity. A redesign of the sample holders to increase the spacing between samples should reduce cross contamination between samples.

This work was supported in part by the Office of Naval Research, The National Science Foundation, The Robert A. Welch Foundation, the State of Texas Advanced Technology Program, and the University of North Texas. Thanks are due to Jana Julien of TI, Inc. for the SIMS measurements.

'R. A. Muller, Science 196, 480 (1977).

${ }^{2}$ K. H. Purser, R. B. Liebert, A. E. Litherland, R. P. Beukens, H. E. Gove, C. L. Bennett, M. R. Glover, and W. E. Sondheim, Rev. Appl. Phys. 12, 1487 (1977)

${ }^{3}$ D. Elmore and F. M. Phillips, Science 236, 543 (1987).

${ }^{4}$ J. R. Davis, A. Rohatgi, R. H. Hopkins, P. D. Blais, P. Rai-Choudhury, J. R. McCormick, and H. C. Mollenkopf, IEEE Trans. Electron. Devices ED-27, 677 (1980).

${ }^{5}$ J. M. Anthony, D. J. Donahue, and T. H. Zabel, Nucl. Instrum. Methods Phys. Res. B 10/11, 498 (1985).

${ }^{6}$ F. D. McDaniel, J. M. Anthony, J. F. Kirchhoff, D. K. Marble, Y. D. Kim, S. N. Renfrow, E. C. Grannan, E. R. Reznik, G. Vizkelethy, and S. Matteson, Nucl. Instrum. Methods Phys. Res. B 89, 242 (1994).

${ }^{7}$ J. F. Kirchhoff, D. K. Marble, D. L. Weathers, F. D. McDaniel, S. Matteson, J. M. Anthony, R. L. Beavers, and T. J. Bennett, Rev. Sci. Instrum. 65, 1570 (1994)

${ }^{8}$ R. G. Wilson, F. A. Stevie, and C. W. Magee, Secondary Ion Mass Spectrometry (Wiley, New York, 1989).

${ }^{9}$ D. L. Weathers, F. D. McDaniel, S. Matteson, J. L. Duggan, J. M. Anthony, and M. A. Douglas, Nucl. Instrum. Methods Phys. Res. B 56/57, 889 (1991).

${ }^{10}$ W. Vandervorst and F. R. Shepherd, J. Vac. Sci. Technol. A 5, 313 (1987).

${ }^{11}$ S. A. Datar, S. N. Renfrow, J. M. Anthony, and F. D. McDaniel, Mater. Res. Soc. Symp. Proc. 406, 395 (1996).

${ }^{12}$ S. A. Datar, S. N. Renfrow, B. N. Guo, J. M. Anthony, Z. Y. Zhao, and F. D. McDaniel, Nucl. Instrum. Methods Phys. Res. B 123, 571 (1997).

${ }^{13}$ J. C. C. Tsai, in VLSI Technology, edited by S. M. Sze (McGraw-Hill, New York, 1988), p. 293. 